\title{
Dinâmica da paisagem no entorno de uma usina hidrelétrica no estado do Paraná, Brasil
}

\section{Landscape dynamics around a hydropower plant in Paraná state, Brazil}

\author{
Amanda Köche Marcon ${ }^{1}$ (D), Franklin Galvão ${ }^{1}$ (D), Renan Augusto Miranda Matias ${ }^{2}$ (D), \\ Christopher Thomas Blum ${ }^{1}$ (D), Paulo Cesar Botosso ${ }^{3}$ (D), Kelly Geronazzo Martins ${ }^{4}$ (1) \\ ${ }^{1}$ Universidade Federal do Paraná - UFPR, Curitiba, SC, Brasil \\ 2Universidade de Brasília - UnB, Brasília, DF, Brasil \\ ${ }^{3}$ EMBRAPA Florestas, Colombo, PR, Brasil \\ ${ }^{4}$ Universidade Estadual do Centro-Oeste - UNICENTRO, Irati, PR, Brasi
}

Como citar: Marcon, A. K., Galvão, F., Matias, R. A. M., Blum, C. T., Botosso, P. C., Martins, K. G. (2020). Dinâmica da paisagem no entorno de uma usina hidrelétrica no estado do Paraná, Brasil. Scientia Forestalis, 48(126), e3278. https://doi.org/10.18671/scifor.v48n126.18

\begin{abstract}
Resumo
O desmatamento e a fragmentação de ambientes naturais são um dos principais fatores responsáveis pelo aumento das concentrações de $\mathrm{CO}_{2}$ na atmosfera. A construção de um grande empreendimento como uma usina hidrelétrica pode alterar a dinâmica da paisagem, impulsionando o crescimento de centros urbanos e outros usos do solo com fins produtivos e econômicos. Neste estudo, foi avaliada a dinâmica da paisagem no entorno da Usina Hidrelétrica Governador Bento Munhoz da Rocha Netto localizada no município de Pinhão, estado do Paraná. Para isto, foram obtidas imagens de satélite dos anos de 1976, 1984 e 2016, contemplando as modificações na paisagem do entorno desde a construção da hidrelétrica, em 1980. Foram calculadas métricas de paisagem de composição, forma, área núcleo, conectividade e isolamento dos fragmentos florestais. Também foram avaliadas métricas de análise temporal do perfil de conversão. Os resultados demonstram uma redução das áreas de floresta em $18,9 \%$, com expressivo desenvolvimento de povoamentos florestais. Houve um grande aumento no número de remanescentes florestais pequenos, indicando um alto grau de fragmentação da paisagem, com redução expressiva das áreas núcleo. Os indicadores temporais do perfil do desmatamento revelaram que as taxas de transformação foram baixas ao longo do tempo, indicando conversões graduais da paisagem. Por outro lado, as conversões das áreas naturais ocorreram, em quase metade da paisagem, nos anos iniciais - que compreendem o período imediato após a construção da usina hidrelétrica. As modificações na paisagem podem estar relacionadas com a construção da usina hidrelétrica, mas o ciclo econômico natural da região pode ter impulsionado a fragmentação dos remanescentes florestais.
\end{abstract}

Palavras-chave: Ecologia de paisagem; Perfil do desmatamento; Fragmentação florestal; Mudanças climáticas.

\begin{abstract}
Deforestation and fragmentation are two of the main factors responsible for climate change. The construction of large enterprises such as hydropower plants can modify landscape dynamics, boosting the urban centers and other economic land uses. We evaluated the landscape around Governador Bento Munhoz da Rocha Netto Hydropower Plant, Paraná, Brazil, aiming to identify the land use dynamics since its construction in 1980. Satellite images of the years 1976, 1984 and 2016 were obtained and landscape and patch metrics of composition, shape, core area, connectivity and isolation were calculated. The deforestation conversion profile was also evaluated. Our results showed a reduction of forest areas by $18.9 \%$, with great replacement of forest stands. A large increase of small forest
\end{abstract}

Fonte de financiamento: Conselho Nacional de Desenvolvimento Científico e Tecnológico, número do processo 140849/2015-7.

Conflito de interesse: Nada a declarar.

Autor correspondente: amandakoche@gmail.com

Recebido: 20 dezembro 2018.

Aceito: 10 julho 2019 .

Editor: Francides Gomes Silva Júnior

(i) Este é um artigo publicado em acesso aberto (Open Access) sob a licença Creative Commons Attribution, que permite uso, distribuição e reprodução c) em qualquer meio, sem restrições desde que o trabalho original seja corretamente citado. 
remnants was noted, indicating a high degree of landscape fragmentation and an expressive reduction of the core area. The deforestation profile revealed that the transformation rates were low, indicating gradual conversions. On the other hand, conversions of natural areas occurred in almost half the area in the initial years - which comprise the period after hydropower plant construction. Changes in the landscape may be related to the hydroelectric plant construction. However, the local economic cycle that happened naturally may have driven the forest fragmentation.

Keywords: Landscape ecology; Deforestation profile; Forest fragmentation; Climate change.

\section{INTRODUÇÃO}

A remoção deliberada dos ambientais naturais é uma das formas mais antigas e significativas pelas quais os seres humanos modificam o meio ambiente (Goudie, 2013; Lausch \& Herzog, 2002). O crescimento das populações humanas e a consequente expansão de suas atividades têm alterado as paisagens naturais em todo o globo terrestre, modificando a organização dos elementos espaciais urbanos e rurais (Tabarelli et al., 2012; Trevisan \& Moschini, 2015).

A fragmentação de ambientes naturais é uma das mais importantes e difundidas consequências da atual dinâmica de uso da terra, pois a taxa de alteração antrópica de paisagens é muito maior do que ocorreria naturalmente nos ecossistemas (Tabarelli \& Gascon, 2005). A conversão de áreas de florestas tropicais para outros usos inevitavelmente implica em mudanças no funcionamento dos ecossistemas (Salati \& Nobre, 1992). Elas alteram o fluxo de energia (Bonan, 1999), podendo emitir ou remover gás carbônico $\left(\mathrm{CO}_{2}\right)$ da atmosfera, além de modificar o balanço de radiação e o albedo da superfície (Marland et al., 2003). Ainda, mudanças na superfície da terra podem modificar os fluxos de calor sensível e latente para a atmosfera, e, portanto, a distribuição de energia dentro do sistema climático, podendo mudar o clima em escala local, regional e até global (Marland et al., 2003).

Do mesmo modo, a inserção de grandes represas, como ocorre quando usinas hidrelétricas são construídas, também resultam em mudanças climáticas em consequência da emissão de gases de efeito estufa decorrentes da vegetação atingida pelo lago artificial (Fearnside, 2005). Os grandes lagos artificiais podem alterar o balanço de carbono, por acumularem e emitirem grandes quantidades de $\mathrm{CO}_{2}$, pela decomposição de matéria orgânica acima da lâmina d'água e liberarem metano $\left(\mathrm{CH}_{4}\right)$ em função da decomposição sob condições anaeróbias, no fundo do reservatório (Fearnside, 2008; Tranvik et al., 2009). As grandes lâminas d'água podem modificar o clima também em função da maior umidade do ar, alterando a formação de nuvens, a precipitação e a evaporação (Williamson et al., 2009). Além disso, a construção de empreendimentos pode alterar a dinâmica do uso do solo como um todo, impulsionando o crescimento de centros urbanos, agropecuária, indústrias e outros fins produtivos (Bortoleto, 2001; Simas \& Pacca, 2013).

Dentro deste contexto, considerando uma paisagem alterada concomitantemente à construção da Usina Hidrelétrica Governador Bento Munhoz da Rocha Netto, o objetivo deste estudo foi avaliar as mudanças no uso dos solos ocorridas desde sua finalização, em 1980. Pretendeu-se responder as seguintes questões: i) Como ocorreu a fragmentação na área ao longo do tempo? ii) Quais as modificações na estrutura da paisagem durante o período avaliado? iii) As modificações na paisagem podem estar relacionadas à construção da usina hidrelétrica?

\section{MATERIAL E MÉTODOS}

\section{Área de estudo}

O estudo foi realizado no entorno da Usina Hidrelétrica Governador Bento Munhoz da Rocha Netto (UHE GBM), localizada no rio Iguaçu, município de Pinhão, Paraná. A construção da barragem foi concluída em dezembro de 1979 e o início das operações se deu em 1980 (COPEL, 2019). 
A altitude na região varia de 700 a 1.250 metros sobre o nível do mar. A formação geológica predominante da região é a Formação Serra Geral e os solos com maior ocorrência na região são Latossolos, Nitossolos e Cambissolos (Bhering \& Santos, 2008; Minerais do Paraná, 2006). O clima é caracterizado por verões brandos e invernos com ocorrência de geadas, com chuvas bem distribuídas ao longo do ano (Cfb) (Alvares et al., 2013). A precipitação média anual no período é de $1.745 \mathrm{~mm}$, variando entre 1.150 e $3.000 \mathrm{~mm}$; a temperatura média do mês mais frio fica abaixo de $14^{\circ} \mathrm{C}$ e a temperatura média no mês mais quente abaixo de $23^{\circ} \mathrm{C}$ (Sistema Nacional de Informações sobre Recursos Hídricos, 2017). A vegetação é constituída majoritariamente por Floresta Ombrófila Mista (Floresta com Araucária), com alguns componentes da Floresta Estacional Semidecidual (Miranda, 2009).

\section{Conjunto de dados}

Foi avaliada a dinâmica espaço-temporal da paisagem com base nos anos 1976, 1984 e 2016, a partir de métricas utilizadas na ecologia de paisagens (Tabela 1). Estas métricas possibilitam diagnosticar e monitorar as mudanças na cobertura da terra e os seus efeitos nos processos ecológicos subjacentes (Ferraz et al., 2009). Para a análise da dinâmica espaço-temporal, a área de estudo teve limites definidos pelo leito maior da represa da UHE GBM, acrescidos $10 \mathrm{~km}$ de bordadura (buffer) (Figura 1). A área total avaliada foi de 222.449,9 ha.

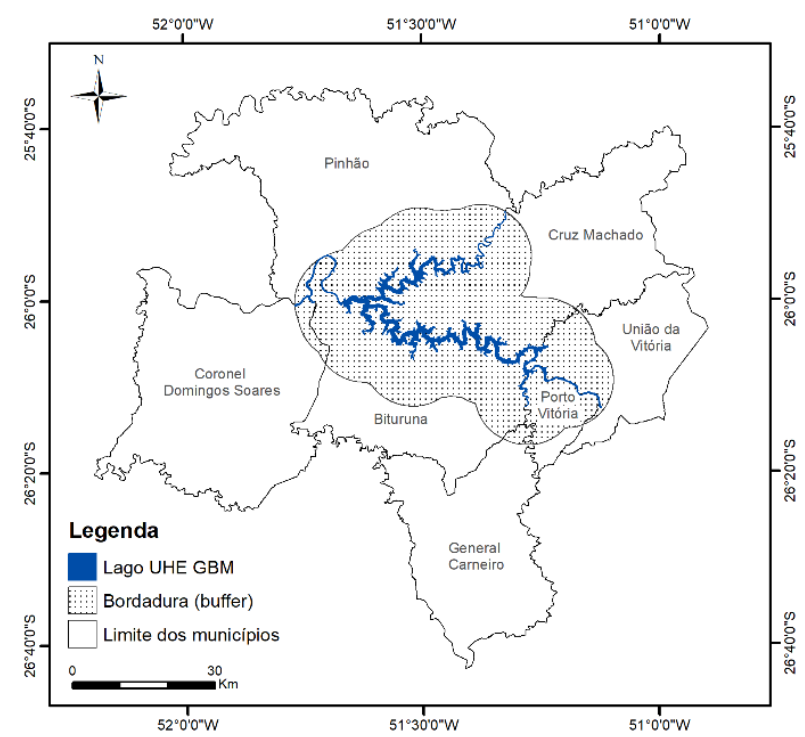

Figura 1. Localização da área de estudo delimitada a partir da bordadura (buffer) da área do lago da Usina Hidrelétrica Gov. Bento Munhoz da Rocha Neto (Fuso 22 S, meridiano central -51).

Para análise da dinâmica do uso dos solos, foram obtidas imagens orbitais pelo site United States Geological Survey (United States Geological Survey, 2018) nos anos de 1976, 1984 e 2016, referentes aos satélites LANDSAT 2, sensor MSS, LANDSAT 5, sensor TM e LANDSAT 8, sensor OLI, respectivamente. As imagens foram submetidas à classificação supervisionada, utilizando o método da máxima verossimilhança. Para avaliação da dinâmica do uso dos solos foram adotadas as classes: floresta (fragmentos de Floresta Ombrófila Mista), povoamento florestal, uso agropastoril, corpos d'água e área urbana. Todo o processamento de dados foi realizado utilizando o software ENVI 5.3 (Harris Geospatial Solutions Inc.).

\section{Dinâmica e estrutura espaço-temporal da paisagem}

As mudanças de uso dos solos e estrutura da paisagem foram avaliadas por métricas calculadas no programa computacional Fragstats (McGarigal et al., 2015). Foram avaliadas métricas de área, forma, área núcleo, conectividade e isolamento, em nível de mancha, classe 
e paisagem (Tabela 1). A descrição detalhada das métricas pode ser visualizada em McGarigal et al. (2015).

Tabela 1. Métricas de paisagem utilizadas neste estudo (Ferraz, 2018; McGarigal et al., 2015).

\begin{tabular}{|c|c|c|c|c|}
\hline \multicolumn{2}{|c|}{ Métrica } & \multirow{2}{*}{$\begin{array}{c}\text { Descrição } \\
\text { Área total (ha) }\end{array}$} & \multicolumn{2}{|l|}{ Fórmula } \\
\hline \multirow[t]{3}{*}{ Área } & PLAND & & $P L A N D=P_{i}=\frac{\sum_{j}^{n} a_{i j}}{A}(100)$ & $\begin{array}{l}\mathrm{P}_{\mathrm{i}} \text { : proporção } \\
\text { ocupada pela } \\
\text { classe na } \\
\text { paisagem; } \mathrm{a}_{\mathrm{ij}} \text { : } \\
\text { área da classe; } \\
\text { A: área total da } \\
\text { paisagem }\left(\mathrm{m}^{2}\right)\end{array}$ \\
\hline & LPI & Porcentager & n coberta pela maior mancha (\%) & \\
\hline & NM & & Número de manchas & \\
\hline Forma & FRAC & $\begin{array}{l}\text { Índice de } \\
\text { dimensão } \\
\text { fractal }\end{array}$ & $F R A C=\frac{2 \ln .25 p_{i j}}{\ln a_{i j}}$ & $\begin{array}{l}\text { pij: }_{\text {ij }} \text { perímetro }(\mathrm{m}) \\
\text { da mancha } \mathrm{ij} ; \\
\mathrm{a}_{\mathrm{ij}}: \text { área }\left(\mathrm{m}^{2}\right) \mathrm{da} \\
\text { mancha ij }\end{array}$ \\
\hline Área núcleo & CORE & $\begin{array}{l}\text { Área núcleo } \\
\text { (ha) }\end{array}$ & CORE $=a_{i j}^{c}\left(\frac{1}{10000}\right)$ & $\begin{array}{l}a_{i j}{ }^{c}=\text { área do } \\
\text { núcleo }\left(\mathrm{m}^{2}\right) \mathrm{da} \\
\text { mancha ij com } \\
\text { base no }\end{array}$ \\
\hline \multirow[t]{2}{*}{$($ borda $=50 \mathrm{~m})$} & TCA & $\begin{array}{l}\text { Total de área } \\
\text { núcleo (ha) }\end{array}$ & $T C A=\sum_{j=1}^{n} a_{i j}^{c}\left(\frac{1}{10000}\right)$ & $\begin{array}{l}\text { tamanho de } \\
\text { borda }(\mathrm{m}) \\
\text { A = área total da } \\
\text { paisagem }\left(\mathrm{m}^{2}\right)\end{array}$ \\
\hline & CPLAND & $\begin{array}{l}\text { Porcentagem de } \\
\text { área núcleo (\%) }\end{array}$ & $C P L A N D=\frac{\sum_{j=1}^{n} a_{i j}^{c}}{A}(100)$ & \\
\hline \multirow{2}{*}{$\begin{array}{l}\text { Conectividade } \\
\text { e proximidade }\end{array}$} & PLAND & $\begin{array}{l}\text { Porcentagem } \\
\text { de área total } \\
\qquad(\%)\end{array}$ & $P L A N D=P_{i}=\frac{\sum_{j}^{n} a_{i j}}{A}(100)$ & $\begin{array}{l}\mathrm{P}_{\mathrm{i}:} \text { proporção } \\
\text { ocupada pela } \\
\text { classe na } \\
\text { paisagem; } \mathrm{a}_{\mathrm{ij}} \text { : } \\
\text { área da classe; } \\
\text { A: área total da } \\
\text { paisagem }\left(\mathrm{m}^{2}\right)\end{array}$ \\
\hline & PROX & $\begin{array}{l}\text { Índice de } \\
\text { proximidade }\end{array}$ & $P R O X=\sum_{g=1}^{n} \frac{a_{i j g}}{h_{i j s}^{2}}$ & $\begin{array}{c}\text { aijs=área da } \\
\text { mancha (m2); hijs } \\
=\text { distância }(\mathrm{m}) \\
\text { entre manchas } \\
\text { da mesma classe } \\
\text { dentro do raio } \\
\text { especificado }(\mathrm{m})\end{array}$ \\
\hline \multirow[t]{3}{*}{$($ raio $=1000 \mathrm{~m})$} & ENN & \multicolumn{2}{|c|}{ Distância euclidiana do vizinho mais próximo } & \\
\hline & CONNECT & $\begin{array}{l}\text { Conectividade } \\
\text { entre } \\
\text { fragmentos }\end{array}$ & CONNECT $=\left[\frac{\sum_{j=k}^{n} c_{i j k}}{\frac{n_{i}\left(n_{i}-1\right)}{2}}\right](100)$ & $\begin{array}{c}\text { Cijk: conexões entre }_{\text {a mancha j e } \mathrm{k}} \\
\text { a }(0=\text { sem conexão; } \\
1=\text { conectado }) ; \\
\mathrm{n}_{\mathrm{i}}=\text { número de } \\
\text { manchas na } \\
\text { paisagem da classe }\end{array}$ \\
\hline & CLUMPY & & Índice de agregação & \\
\hline $\begin{array}{l}\text { Agregação e } \\
\text { justaposição }\end{array}$ & اנا & $\begin{array}{l}\text { Índice de } \\
\text { dispersão e } \\
\text { justaposição }\end{array}$ & $I J I=\frac{-\sum_{k=i}^{m}\left[\left(\frac{e_{i k}}{\sum_{k=1}^{m} e_{i k}}\right) \ln \left(\frac{e_{i k}}{\sum_{k=1}^{m} e_{i k}}\right)\right]}{\ln (m-1)}(100)$ & $\begin{array}{c}\text { e } \mathrm{e}_{\mathrm{ik}} \text { : comprimento } \\
\text { (m) da borda entre } \\
\text { classes; m: número } \\
\text { de fragmentos da } \\
\text { classe }\end{array}$ \\
\hline
\end{tabular}


Tabela 1. Continuação...

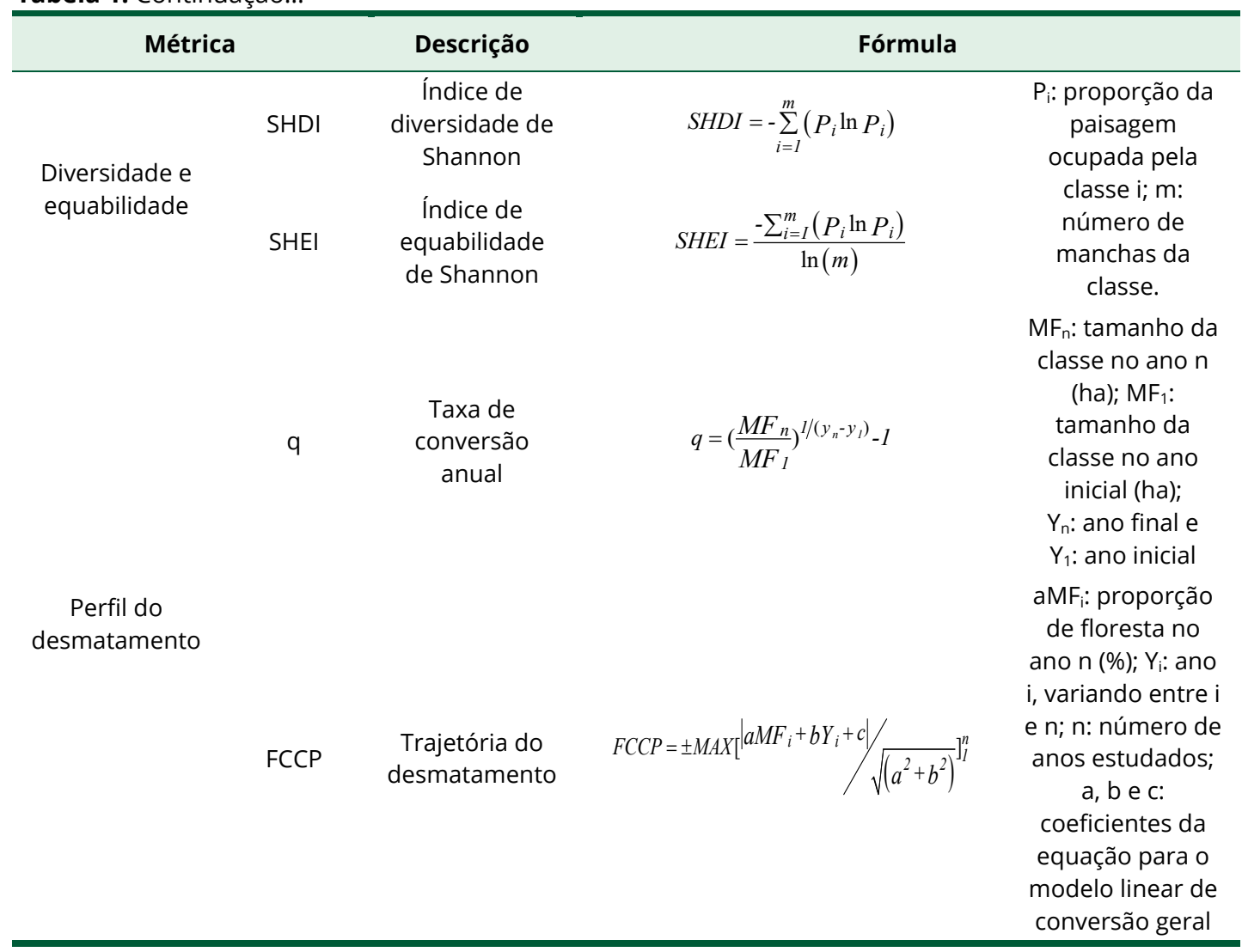

Para investigar se as modificações na paisagem foram relacionadas à construção da UHE GBM, foi realizada uma análise temporal do perfil de conversão, utilizando o pacote Land-Use Change Analysis Tools (LUCAT) (Ferraz, 2018), do software ESRI ArcGIS versão 9.3. A taxa de conversão anual $(q)$ e a trajetória do desmatamento (FCCP) foram utilizados como indicadores temporais do desmatamento. Valores negativos de $q$ representam redução na área ocupada, e positivos representam aumento (Ferraz et al., 2009). Para o indicador FCCP, valores positivos indicam que a conversão se concentra nos anos iniciais, valores negativos, nos anos finais, valores menores indicam padrões de conversão dispersos entre os períodos, enquanto valores elevados representam mudanças drásticas na paisagem (Ferraz et al., 2009). Para esta análise, as classes agropastoril, área urbana, povoamento florestal e a área que compreende o lago artificial da usina foram agrupadas em uma única classe, denominada áreas antrópicas. A área em estudo foi subdividida em 9.150 quadrantes de 25 hectares numerados, seguindo as recomendações de Ferraz (2018), utilizando a extensão Hawth's Analysis Tools (SpatialEcology).

Para a verificação das alterações ocorridas na paisagem foram aplicados nas métricas testes estatísticos de normalidade e de postos de Kruskal-Wallis utilizando o software $\mathrm{R}$ (R Development Core Team, 2017).

\section{RESULTADOS E DISCUSSÃO}

\section{Dinâmica e estrutura da paisagem}

Seguindo a tendência global da alarmante redução das áreas naturais (Bradshaw et al., 2007), a paisagem no entorno da UHE GBM sofreu uma diminuição nas áreas de floresta numa ordem de 18,9\% (Figura 2, Tabela 2). Foi detectado um aumento em área das classes "corpos d'água", "povoamentos florestais" e "áreas urbanas", demonstrando que o empreendimento pode ter contribuído no desenvolvimento dos municípios adjacentes. O aumento em área dos corpos d'água está relacionado com a construção do reservatório da UHE GBM. 

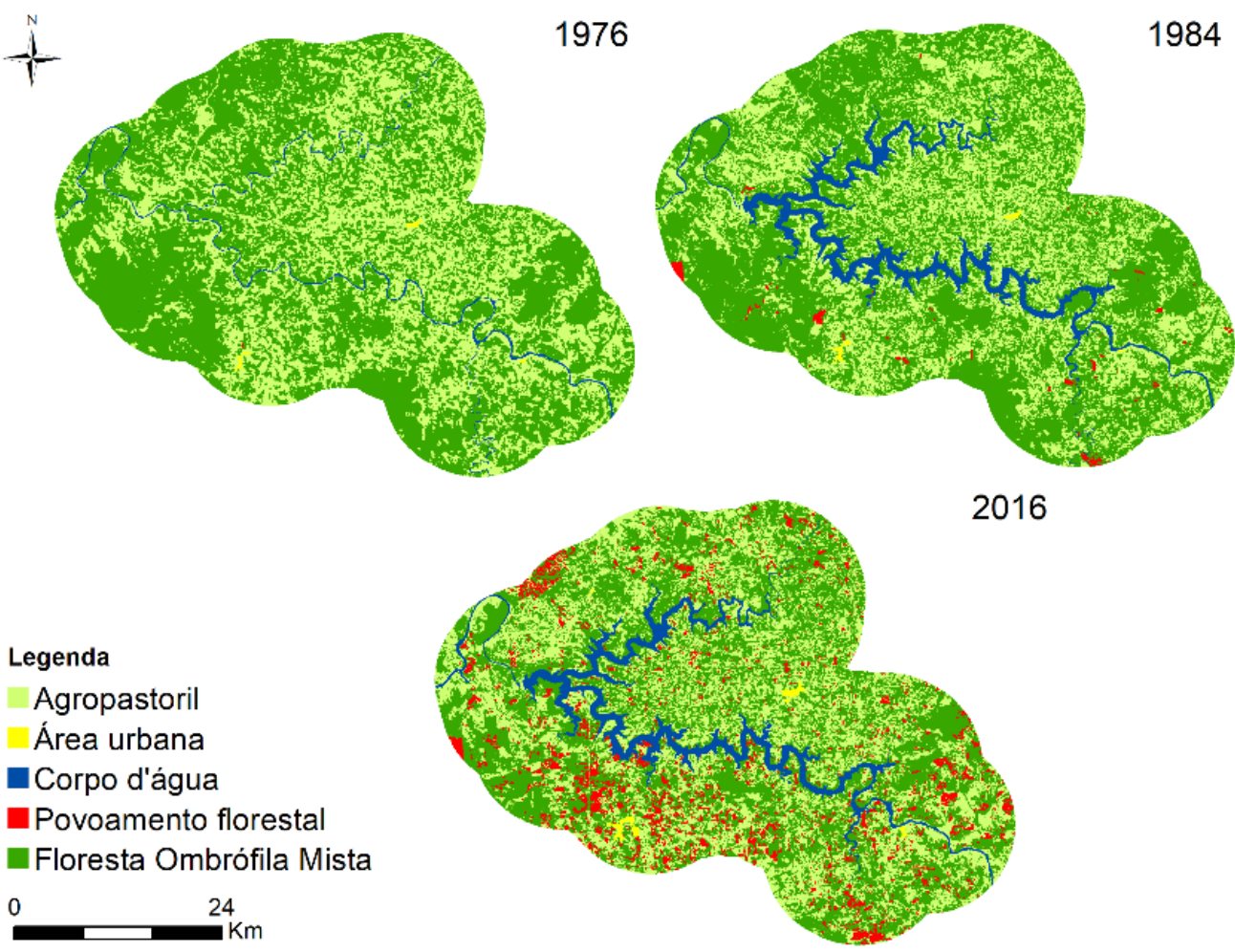

Figura 2. Uso do solo do entorno da UHE GBM, Paraná, Brasil, para os anos de 1976, 1984 e 2016.

Tabela 2. Uso do solo em área (ha), porcentagem da área total (\%), número de manchas (NM) e percentual da paisagem ocupada pelo maior polígono (LPI).

\begin{tabular}{|c|c|c|c|c|c|c|c|c|c|c|c|c|}
\hline \multirow{2}{*}{ Classes } & \multicolumn{3}{|c|}{ Área (ha) } & \multicolumn{3}{|c|}{ Área (\%) } & \multicolumn{3}{|c|}{ NM } & \multicolumn{3}{|c|}{ LPI (\%) } \\
\hline & 1976 & 1984 & 2016 & 1976 & 1984 & 2016 & 1976 & 1984 & 2016 & 1976 & 1984 & 2016 \\
\hline Agropastoril & 85.741 & 78.94 & 83.121 & 38,5 & 35,5 & 37,3 & 1.683 & 3.488 & 4.108 & 13,9 & 6,5 & 4,6 \\
\hline Corpos d'água & 2.95 & 13.328 & 13.166 & 1,3 & 6,0 & 5,9 & 68 & 104 & 75 & 0,3 & 5,7 & 5,6 \\
\hline Floresta & 133.521 & 128.715 & 108.315 & 60,0 & 57,9 & 48,7 & 992 & 2.351 & 3.554 & 31,9 & 22,3 & 9,4 \\
\hline $\begin{array}{l}\text { Povoamento } \\
\text { florestal }\end{array}$ & 12 & 1.171 & 17.19 & 0,0 & 0,5 & 7,7 & 1 & 104 & 3.63 & 0,0 & 0,1 & 0,1 \\
\hline Área urbana & 225 & 295 & 656 & 0,1 & 0,1 & 0,3 & 3 & 4 & 8 & 0,0 & 0,1 & 0,2 \\
\hline Total & & $222.449,4$ & & & & & 2.747 & 6.051 & 11.375 & & & \\
\hline
\end{tabular}

A matriz da paisagem permaneceu florestal, ainda que reduzida de 60 para $48,7 \%$. Vidolin et al. (2011) avaliaram a estrutura da paisagem em uma região próxima a este estudo, nos municípios de General Carneiro, Palmas e Bituruna e também encontraram uma matriz de cobertura do solo predominantemente florestal. A região possui uma boa continuidade florestal, o que pode favorecer a conexão entre os remanescentes de florestas nativas (Vidolin et al., 2011).

Embora a matriz seja florestal, é importante avaliar a sua permeabilidade, ou o quanto ela possibilita um efetivo contato entre fragmentos no sentido do fluxo biológico (Metzger, 2006). Como a paisagem se fragmentou muito com o passar dos anos, ainda que a área total florestada não tenha reduzido drasticamente, ocorreu um grande aumento no grau de retalhamento. Uma mancha habitat é mais propensa a ser visitada pela fauna se possuir no seu entorno muitos fragmentos de áreas naturais, do que se possuir manchas esparsas (Hein et al., 2004). As áreas muito fragmentadas, no entanto, possuem um mosaico complexo de diversas classes de uso dos solos, que podem diferir em sua resistência ao movimento de indivíduos entre os fragmentos, sendo mais ou menos efetivamente isolados (Ricketts, 2001). 
Houve um aumento nos índices de diversidade (SHDI) e equabilidade (SHEI) com o passar dos anos (Tabela 3). O índice SHDI é maior à medida que aumenta a diversidade de manchas (McGarigal et al., 2015). De maneira análoga, o índice SHEl se aproxima de um quando há uma distribuição uniforme da área entre as classes, resultando em maior uniformidade (McGarigal et al., 2015). O aumento nesses índices foi resultante da melhor distribuição da área entre as classes, como consequência da redução da classe floresta. A fragmentação dos ambientes naturais promoveu a expansão de outras formas de uso da terra, criando fronteiras entre elas e aumentando a heterogeneidade do mosaico (Metzger, 2006). Outros estudos de dinâmica espaço-temporal da paisagem realizados na Mata Atlântica verificaram o mesmo padrão (Pereira et al., 2017; Pirovani et al., 2014), reiterando a grave situação de degradação deste bioma (Morellato \& Haddad, 2000; Ribeiro et al., 2009).

Tabela 3. Métricas da paisagem do entorno da UHE GBM, para os anos de referência.

\begin{tabular}{ccccc}
\hline & Métricas & $\mathbf{1 9 7 6}$ & $\mathbf{1 9 8 4}$ & $\mathbf{2 0 1 6}$ \\
\hline LPI & Porcentagem da área ocupada pelo maior fragmento (\%) & 31,9 & 22,3 & 9,4 \\
TCA & Total de área núcleo (ha) & 162.598 & 143.983 & 125.880 \\
SHDI & Índice de diversidade de Shannon (SHDI $\geq 0$, sem limite) & 0,74 & 0,89 & 1,10 \\
SHEI & Índice de equabilidade de Shannon (0 $\leq$ SHEI $\leq 1)$ & 0,46 & 0,55 & 0,68 \\
\hline
\end{tabular}

As métricas específicas da classe floresta demonstram uma redução em sua área total, e consequentemente nas áreas núcleo, em função da fragmentação dos remanescentes (Figura 3, tabela 4). A redução do tamanho e a alteração da forma dos fragmentos podem influenciar inúmeros processos ecológicos importantes, principalmente pelas suas relações com o efeito de borda (Herrmann et al., 2005). O tamanho da mancha é um dos principais preditores da riqueza de espécies em uma área natural (Drinnan, 2005; Lamberson et al., 1994). Grandes fragmentos mantêm um bom subconjunto de espécies, mas aqueles com área reduzida possuem poucas espécies, geralmente as mais generalistas, que conseguem sobreviver em um ambiente altamente influenciado pelo entorno (Farina, 2008; Martensen et al., 2012). Quanto menor e mais irregular o fragmento, maior será, proporcionalmente, a área sobre influência do ecossistema circundante (Hill \& Curran, 2003). Nas regiões de borda dos fragmentos, geralmente as condições são de maior temperatura, menor umidade do ar e do solo, maior intensidade luminosa e de ventos, influenciando a composição e distribuição da comunidade biológica (Laurance, 2000; Murcia, 1995). Especialmente as espécies que só ocorrem no interior das florestas podem sofrer um declínio populacional (Bender et al., 1998).
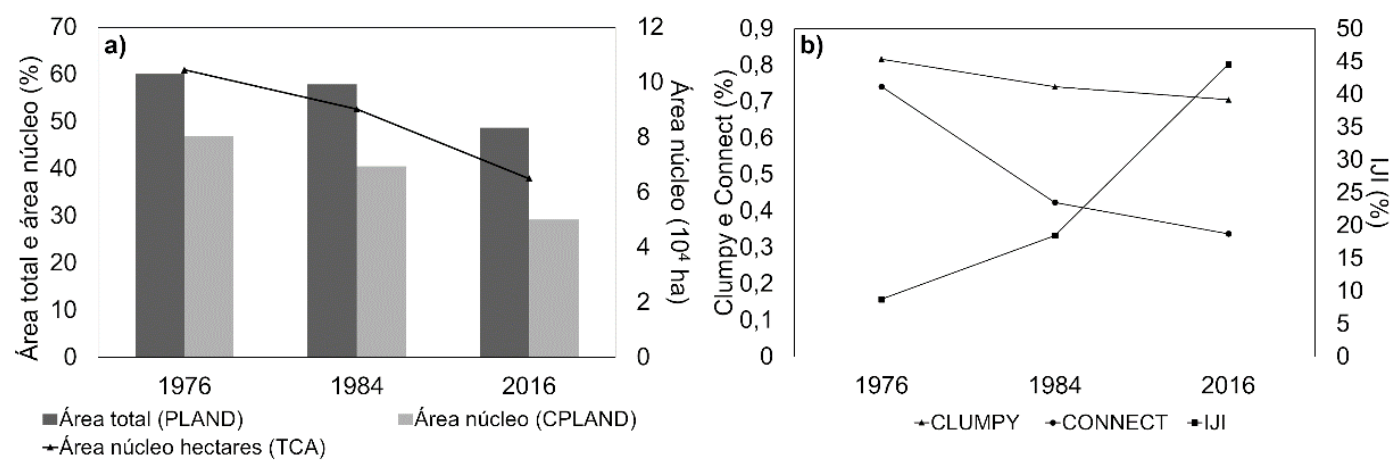

Figura 3. Métricas da classe floresta para os anos de referência. a) Métricas de área; b) Métricas de isolamento e conectividade. PLAND: porcentagem da área total; CPLAND: porcentagem das áreas

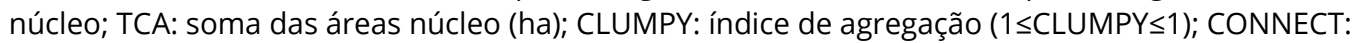
conectividade entre fragmentos ( $0 \leq C O N N E C T \leq 100)$; IJ: índice de dispersão e justaposição $(0<|J| \leq 100)$. 
Tabela 4. Métricas de forma, área núcleo e proximidade para os fragmentos da classe floresta para os anos de referência.

\begin{tabular}{|c|c|c|c|c|c|c|}
\hline \multicolumn{3}{|c|}{ Métrica } & 1976 & 1984 & 2016 & $p^{*}$ \\
\hline \multirow{4}{*}{ 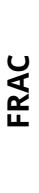 } & \multirow{4}{*}{$\begin{array}{c}\text { Índice de } \\
\text { dimensão } \\
\text { fractal } \\
(1 \leq \text { FRAC } \leq 2)\end{array}$} & média & 1,050 & 1,043 & 1,046 & $<0,001$ \\
\hline & & mediana & 1,038 & 1,031 & 1,031 & \\
\hline & & desvio padrão & 0,047 & 0,048 & 0,053 & \\
\hline & & CV (\%) & 4,447 & 4,627 & 5,115 & \\
\hline \multirow{4}{*}{ 亗 } & \multirow{4}{*}{$\begin{array}{c}\text { Área central } \\
\text { total dos } \\
\text { fragmentos (ha) }\end{array}$} & média & 105,313 & 38,347 & 18,286 & $<0,001$ \\
\hline & & mediana & 0,750 & 0,000 & 0,000 & \\
\hline & & desvio padrão & $1.931,748$ & 928,199 & 321,288 & \\
\hline & & CV (\%) & $1.834,292$ & $2.420,526$ & $1.757,043$ & \\
\hline \multirow{4}{*}{ 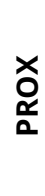 } & \multirow{4}{*}{$\begin{array}{c}\text { Índice de } \\
\text { proximidade } \\
(P R O X \geq 0)\end{array}$} & média & $13.229,676$ & $14.449,856$ & $3.793,465$ & $<0,001$ \\
\hline & & mediana & $4.951,845$ & $7.948,761$ & $1.304,547$ & \\
\hline & & desvio padrão & $18.370,380$ & $15.548,264$ & $5.265,286$ & \\
\hline & & CV (\%) & 138,857 & 107,602 & 138,799 & \\
\hline \multirow{4}{*}{$z_{\mathbf{u}}$} & \multirow{4}{*}{$\begin{array}{c}\text { Distância } \\
\text { euclidiana do } \\
\text { vizinho mais } \\
\text { próximo (m) }\end{array}$} & média & 164,404 & 130,684 & 130,453 & $<0,001$ \\
\hline & & mediana & 150,000 & 111,803 & 111,803 & \\
\hline & & desvio padrão & 62,915 & 40,392 & 42,043 & \\
\hline & & CV (\%) & 38,268 & 30,909 & 32,229 & \\
\hline
\end{tabular}

*Valor de $p$ determinado pelo teste de postos de Kruskal-Wallis.

Mesmo fragmentos com mais de 10 ha (89\% dos fragmentos no ano de 2016) sofrem com efeitos de borda significativos (Bradshaw, 1992). Com a redução das manchas de floresta à fragmentos muito pequenos, a área núcleo remanescente em muitos casos tende a zero (Cemin et al., 2009). Por outro lado, estes pequenos fragmentos podem servir como abrigo à fauna para acessar remanescentes maiores (stepping-stones), influenciando as estratégias de fuga de animais como pequenos mamíferos (Farina, 2008).

Embora a forma dos fragmentos de floresta não tenha se alterado substancialmente entre os anos, as métricas de conectividade e proximidade, elementos chave para a manutenção das relações na paisagem (Martensen et al., 2008), indicam uma considerável piora nas inter-relações entre remanescentes. A menor conectividade entre fragmentos reduz as chances das espécies explorarem o mosaico da paisagem e fluxo gênico entre populações, influenciando taxas de colonização e extinção (Martensen et al., 2008; Tischendorf \& Fahrig, 2000). Em alguns casos, a conectividade pode ser ainda mais importante do que o tamanho dos fragmentos, especialmente se a cobertura de áreas naturais está em uma quantidade intermediária (Martensen et al., 2008), como é o caso desta paisagem em 2016 (48,7\%). Além da perda da conectividade pela fragmentação em si, para Silva et al. (2014), em construções de usinas hidrelétricas, os reservatórios eliminam a conectividade dos fragmentos, resultando no isolamento de remanescentes as margens do empreendimento.

\section{Análise temporal do perfil do desmatamento}

O desmatamento na área de estudo segue o padrão observado na maioria das regiões da Mata Atlântica, com áreas naturais sendo convertidas em antrópicas com o passar dos anos. Em relação à taxa de conversão anual, o índice $q$ apresentou taxas entre -100 a $5 \% \mathrm{ano}^{-1}$, mostrando uma grande variação entre os quadrantes, que tiveram diferentes taxas de desmatamento. Taxas de $100 \% \mathrm{ano}^{-1}$, que indicam rápidas conversões na paisagem, foram distribuídas em toda a área, indicando que houve rápido desmatamento logo nos primeiros anos nestes locais, período imediato após a construção da usina hidrelétrica (Figura 4). No entanto, a maioria dos quadrantes $(50,7 \%)$ apresentou zero ou pouca alteração e 39,6\% destes apresentaram taxa de transformação entre -20 a $0 \% \mathrm{ano}^{-1}$, indicando baixas taxas de conversão anual. Os quadrantes com redução de $100 \% \mathrm{ano}^{-1}$ da área florestada representam $9,8 \%$ do total. 


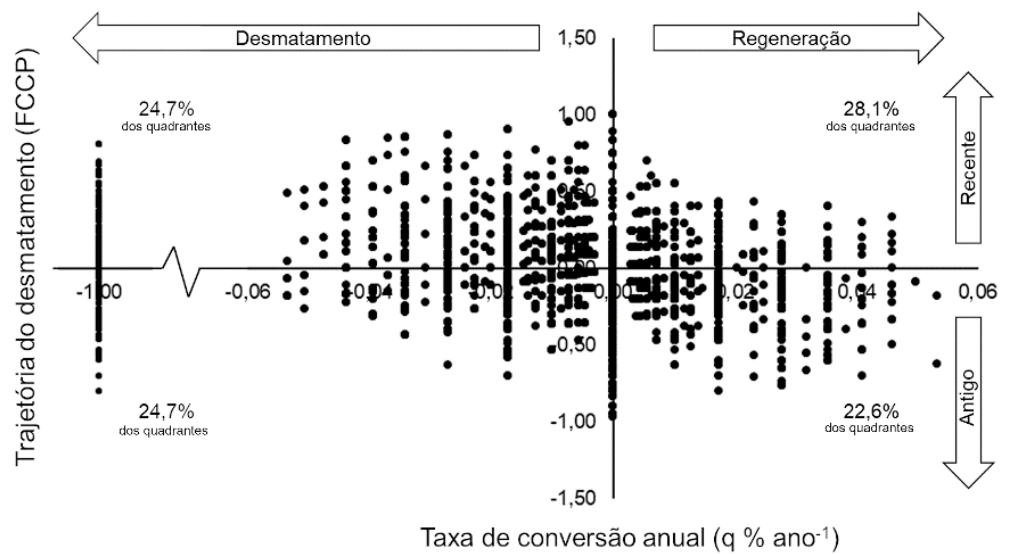

Figura 4. Trajetória do desmatamento (FCCP) e taxa de conversão anual (q) para a paisagem da UHE GBM, entre os anos de 1976 e 2016. As porcentagens dos quadrantes que não se modificaram $\left(q=0 \% a^{-1} o^{-1}\right.$ foram incluídas como regeneração.

O índice que demonstra a trajetória do desmatamento (FCCP) demonstrou que existem basicamente dois diferentes perfis. Em $45,4 \%$ dos quadrantes o valor de FCCP foi negativo, entre $-0,5$ a 0 , indicando conversão nos anos finais. E em $48,9 \%$ dos quadrantes a conversão foi nos anos iniciais (valores positivos, de 0 a 0,5 ). Então, como os valores não foram extremos, para quase toda a área as conversões foram graduais. Somente em $4,8 \%$ da área houve conversões drásticas.

Possivelmente, as conversões nos anos iniciais, que são concomitantes à construção da usina hidrelétrica, foram impulsionadas pelo empreendimento. Em termos históricos, momentos de mudanças na econômica local, como o desenvolvimento urbano e a expansão agropecuária ocasionam altos níveis de fragmentação (Silva et al., 2014; Zaú, 1998). A construção de grandes lagos artificiais também altera os padrões de uso da terra, seja pelo progresso da região em termos industriais e turísticos ou mesmo devido à maior acessibilidade à áreas adjacentes (Wu et al., 2003). Terras agrícolas que eram financeiramente marginais se tornam mais lucrativas, resultando em degradação do habitat florestal remanescente (Lees et al., 2016). Por outro lado, é possível que o desenvolvimento socioeconômico natural da região nesta época, em função da instalação de empresas de base florestal, do aumento no número de empregos diretos e indiretos, entre outros fatores, contribuiu para a fragmentação da paisagem. Parâmetros como o índice de desenvolvimento humano (IDH) e o produto interno bruto (PIB), podem ser tomados como medidas do gradativo progresso na região (Fernandes et al., 2018; Programa das Nações Unidas para o Desenvolvimento, 2019). Para municípios como Pinhão, Bituruna e Cruz Machado, o IDH vem aumentando com o passar dos anos, mudando de 0,36 em 1991, para 0,66 em 2010, em valores médios (Atlas do Desenvolvimento Humano no Brasil, 2019). Quanto ao PIB per capita, também foi verificado um aumento entre os anos de 1999 e 2016, com respectivos valores (R\$/hab.) de 12.385 e 58.776,09 para Pinhão, 4.362 e 17.642,5 para Bituruna e 3.710 e 16.265,07 para Cruz Machado (Instituto Brasileiro de Geografia e Estatística, 2019). Também foi possível detectar um aumento no número de pequenos fragmentos para a classe agropastoril, que pode estar relacionado ao desmembramento de imóveis rurais em propriedades menores, prática constante no interior dos Estados do Brasil (Almeida \& Sardagna, 2000).

A fragmentação parece ser comum nas áreas do entorno de grandes empreendimentos. Zhao et al. (2012), ao avaliar os impactos sobre a paisagem da construção de uma usina hidrelétrica em Pequim, encontraram resultados que sugerem um padrão mais fragmentado e níveis mais altos de diversidade e uniformidade após a construção da barragem, resultado da redução de grandes fragmentos. As alterações na paisagem também ocorreram nos campos naturais na China, segundo o estudo de Ouyang et al. (2010), após 30 anos de funcionamento de uma cascata de usinas. 
Apesar de, em quase metade dos quadrantes, a taxa de conversão anual indique regeneração das áreas naturais (valores positivos), elas foram baixas, alcançando um máximo de regeneração de 5,3\% ano-1. A regeneração de florestas secundárias sobre pastagens abandonadas pode ser resultado do abandono de áreas com baixíssimo potencial de produção, como em encostas íngremes e solos rochosos (Ferraz et al., 2014). Ainda que baixas, estas taxas de regeneração são importantes, pois trazem diversos benefícios ao ecossistema, recuperando, pelo menos parcialmente, suas funcionalidades (Mesquita, 2000; Parrotta et al., 1997).

\section{Considerações sobre o clima local}

As principais modificações observadas na paisagem do entorno da UHE GBM foram a perda de áreas de floresta para povoamentos florestais e para a própria área do reservatório, além da fragmentação. Os maciços florestais fornecem uma variedade de bens e serviços para a sociedade e contribuem para o armazenamento de carbono (Winjum \& Schroeder, 1997). No caso de plantações florestais comerciais, o potencial de imobilização de carbono é superior quando comparado aos sistemas agropecuários (Higa et al., 2017), ainda que seja cerca de $28 \%$ menor do que o das florestas, segundo Liao et al. (2010). Além do maior estoque de carbono do que os sistemas agropastoris, as plantações florestais propiciam um albedo reduzido - mais radiação de ondas curtas é absorvida na superfície - e um aumento da evapotranspiração, por crescerem rapidamente (Findell et al., 2007; Kirschbaum et al., 2011; Swift Junior et al., 1975). Assim sendo, como as características gerais não são bruscamente modificadas com a substituição das florestas por povoamentos florestais - como a cobertura do solo, a renovação do estoque de carbono e as taxas de evapotranspiração - infere-se que não tenham ocorrido alterações relevantes no clima local em decorrência da conversão das florestas naturais. Contudo, outros estudos precisam ser realizados para elucidar os efeitos no clima local decorrentes da inserção de grandes lâminas d'água.

\section{CONCLUSÃO}

Houve grande fragmentação das áreas de floresta ao longo do período analisado, com expressivo aumento no grau de retalhamento da paisagem. Por consequência disso, houve uma redução expressiva das áreas núcleo e da área ocupada pelo maior fragmento florestal. Por outro lado, a distribuição das classes de uso do solo ao longo dos anos avaliados demonstra que não houve uma redução drástica na área total de florestas. Também houve um expressivo aumento das áreas urbanas. As modificações na paisagem podem estar relacionadas com a construção da usina hidrelétrica, visto que empreendimentos desta magnitude certamente impulsionam a economia local. Contudo, o ciclo econômico natural da região provavelmente impulsionou a fragmentação dos remanescentes florestais.

\section{REFERÊNCIAS}

Alvares, C. A., Stape, J. L., Sentelhas, P. C., Moraes Gonçalves, J. L., \& Sparovek, G. (2013). Köppen's climate classification map for Brazil. Meteorologische Zeitschrift (Berlin), 22(6), 711-728. http://dx.doi.org/10.1127/0941-2948/2013/0507.

Almeida, E. C., \& Sardagna, C. D. (2000). O parcelamento do imóvel rural via fração mínima de parcelamento frente à função social da propriedade. Revista de Informação Legis/ativa, 37(146), 209216.

Atlas do Desenvolvimento Humano no Brasil (2019). Recuperado em 24 de julho de 2019, de http://www.atlasbrasil.org.br/2013/pt/

Bender, D. J., Contreras, T. A., \& Fahrig, L. (1998). Habitat loss and population decline: A meta-analysis of the patch size effect. Ecology, 79(2), 517-533. http://dx.doi.org/10.1890/00129658(1998)079[0517:HLAPDA]2.0.CO;2.

Bhering, S. B., \& Santos, H. G. (2008). Mapa de solos do Estado do Paraná, legenda atualizada. Fortaleza: Congresso Brasileiro de Ciência do Solo. 
Bonan, G. B. (1999). Frost followed the plow: Impacts of deforestation on the climate of the United States. Ecological Applications, 9(4), 1305-1315. http://dx.doi.org/10.1890/10510761(1999)009[1305:FFTPIO]2.0.CO;2.

Bortoleto, E. M. (2001). A implantação de grandes hidrelétricas: desenvolvimento, discurso e impactos. Geografares, 2, 53-62.

Bradshaw, C. J. A., Sodhi, N. S., Peh, K. S.-H., \& Brook, B. W. (2007). Global evidence that deforestation amplifies flood risk and severity in the developing world. Global Change Biology, 13(11), 2379-2395. http://dx.doi.org/10.1111/j.1365-2486.2007.01446.x.

Bradshaw, F. J. (1992). Quantifying edge effect and patch size for multiple-use silviculture - a discussion paper. Forest Ecology and Management, 48(3-4), 249-264. http://dx.doi.org/10.1016/03781127(92)90148-3.

Cemin, G., Perico, E., \& Rempel, C. (2009). Composição e configuração da paisagem da sub-bacia do Arroio Jacaré, Vale do Taquari, RS, com ênfase nas áreas de florestas. Revista Árvore, 33(4), 705-711. http://dx.doi.org/10.1590/S0100-67622009000400013.

COPEL (2019). Usina Bento Munhoz da Rocha Netto. Recuperado em 02 de janeiro 2019, de https://www.copel.com/hpcopel/geracao

Drinnan, I. N. (2005). The search for fragmentation thresholds in a Southern Sydney Suburb. Biological Conservation, 124(3), 339-349. http://dx.doi.org/10.1016/j.biocon.2005.01.040.

Farina, A. (2008). Principles and methods in landscape ecology: towards a science of the landscape (Vol. 3, 412 p.). Dordrecht: Springer Science \& Business Media.

Fearnside, P. M. (2005). Do hydroelectric dams mitigate global warming? The case of Brazil's Curuá-Una Dam. Mitigation and Adaptation Strategies for Global Change, 10(4), 675-691. http://dx.doi.org/10.1007/s11027-005-7303-7.

Fearnside, P. M. (2008). Hidrelétricas como "fábricas de metano": o papel dos reservatórios em áreas de floresta tropical na emissão de gases de efeito estufa. Oecologia Brasiliensis, 12(1), 100-115.

Fernandes, R. B., Tavares, A. L., \& Azevedo, Y. G. P. (2018). Relação entre o valor adicionado das atividades econômicas e o produto interno bruto do rio grande do norte. $R A C E, 17(2), 757-782$. http://dx.doi.org/10.18593/race.v17i2.17238.

Ferraz, S. F. B., Ferraz, K. M. P. M. B., Cassiano, C. C., Brancalion, P. H. S., Luz, D. T. A., Azevedo, T. N., Tambosi, L. R., \& Metzger, J. P. (2014). How good are tropical forest patches for ecosystem services provisioning? Landscape Ecology, 29(2), 187-200. http://dx.doi.org/10.1007/s10980-014-9988-z.

Ferraz, S. F. B. (2018). Land-use change analysis tools (LUCAT). Recuperado em 02 de janeiro 2019, de http://www.dokuwiki.Icf.esalq.usp.br/silvio/doku.php?id=extras:extras

Ferraz, S. F., Vettorazzi, C. A., \& Theobald, D. M. (2009). Using indicators of deforestation and land-use dynamics to support conservation strategies: A case study of central Rondônia, Brazil. Forest Ecology and Management, 257(7), 1586-1595. http://dx.doi.org/10.1016/j.foreco.2009.01.013.

Findell, K. L., Shevliakova, E., Milly, P. C. D., \& Stouffer, R. J. (2007). Modeled impact of anthropogenic land cover change on climate. Journal of Climate, 20(14), 3621-3634. http://dx.doi.org/10.1175/JCLI4185.1.

Goudie, A. S. (2013). Human impact on the natural environment: past, present and future (7th ed., 410 p.). United Kingdom: John Wiley \& Sons.

Hein, S., Pfenning, B., Hovestadt, T., \& Poethke, H.-J. (2004). Patch density, movement pattern, and realised dispersal distances in a patch-matrix landscape - A simulation study. Ecological Modelling 174(4), 411-420. http://dx.doi.org/10.1016/j.ecolmodel.2003.10.005.

Herrmann, B. C., Rodrigues, E., \& Lima, A. (2005). A paisagem como condicionadora de bordas de fragmentos florestais. Floresta, 35(1), 13-22. http://dx.doi.org/10.5380/rf.v35i1.2427.

Higa, R. C. V., Zanatta, J. A., \& Rachwal, M. F. G. (2017). Plantações florestais comerciais e a mitigação na mudança do clima. In Y. M. M. Oliveira \& E. B. Oliveira (Eds.), Plantações florestais: geração de benefícios com baixo impacto ambiental (pp. 67-112). Brasília: EMBRAPA.

Hill, J. L., \& Curran, P. J. (2003). Area, shape and isolation of tropical forest fragments: effects on tree species diversity and implications for conservation. Journal of Biogeography, 30(9), 1391-1403. http://dx.doi.org/10.1046/j.1365-2699.2003.00930.x.

Instituto Brasileiro de Geografia e Estatística - IBGE (2019). Produto Interno Bruto dos Municípios. Recuperado em 24 de julho 2019, de https://www.ibge.gov.br/estatisticas/economicas/contasnacionais/9088-produto-interno-bruto-dos-municipios.html?=\&t=downloads. 
Kirschbaum, M. U. F., Whitehead, D., Dean, S. M., Beets, P. N., Shepherd, J. D., \& Ausseil, A.-G. E. (2011). Implications of albedo changes following afforestation on the benefits of forests as carbon sinks. Biogeosciences, 8(12), 3687-3696. http://dx.doi.org/10.5194/bg-8-3687-2011.

Lamberson, R. H., Noon, B. R., Voss, C., \& McKelvey, K. S. (1994). Reserve design for territorial species: the effects of patch size and spacing on the viability of the northern spotted owl*. Conservation Biology, 8(1), 185-195. http://dx.doi.org/10.1046/j.1523-1739.1994.08010185.x.

Laurance, W. F. (2000). Do edge effects occur over large spatial scales? Trends in Ecology \& Evolution, 15(4), 134-135. PMid:10717681. http://dx.doi.org/10.1016/S0169-5347(00)01838-3.

Lausch, A., \& Herzog, F. (2002). Applicability of landscape metrics for the monitoring of landscape change: issues of scale, resolution and interpretability. Ecological Indicators, 2(1-2), 3-15. http://dx.doi.org/10.1016/S1470-160X(02)00053-5.

Lees, A. C., Peres, C. A., Fearnside, P. M., Schneider, M., \& Zuanon, J. A. S. (2016). Hydropower and the future of Amazonian biodiversity. Biodiversity and Conservation, 25(3), 451-466. http://dx.doi.org/10.1007/s10531-016-1072-3.

Liao, C., Luo, Y., Fang, C., \& Li, B. (2010). Ecosystem Carbon stock influenced by plantation practice: implications for planting forests as a measure of climate change mitigation. PLoS One, 5(5), e10867. PMid:20523733. http://dx.doi.org/10.1371/journal.pone.0010867.

Marland, G., Pielke Sr, R. A., Apps, M., Avissar, R., Betts, R. A., Davis, K. J., Frumhoff, P. C., Jackson, S. T., Joyce, L. A., Kauppi, P., Katzenberger, J., MacDicken, K. G., Neilson, R. P., Niles, J. O., Niyogi, D. S., Norby, R. J., Pena, N., Sampson, N., \& Xue, Y. (2003). The climatic impacts of land surface change and carbon management, and the implications for climate-change mitigation policy. Climate Policy, 3(2), 149-157. http://dx.doi.org/10.3763/cpol.2003.0318.

Martensen, A. C., Pimentel, R. G., \& Metzger, J. P. (2008). Relative effects of fragment size and connectivity on bird community in the Atlantic Rain Forest: implications for conservation. Biological Conservation, 141(9), 2184-2192. http://dx.doi.org/10.1016/j.biocon.2008.06.008.

Martensen, A. C., Ribeiro, M. C., Banks-Leite, C., Prado, P. I., \& Metzger, J. P. (2012). Associations of Forest Cover, Fragment Area, and Connectivity with Neotropical Understory Bird Species Richness and Abundance. Conservation Biology, 26(6), 1100-1111. PMid:23003666. http://dx.doi.org/10.1111/j.1523-1739.2012.01940.x.

McGarigal, K., Cushman, S. A., Neel, M. C., \& Ene, E. (2015). FRAGSTATS: Spatial Pattern Analysis Program for Categorical Maps. Amherst: University of Massachusetts.

Mesquita, R. (2000). Management of advanced regeneration in secondary forests of the Brazilian Amazon. Forest Ecology and Management, 130(1-3), 131-140. http://dx.doi.org/10.1016/S03781127(99)00174-7.

Metzger, J. P. (2006). Como lidar com regras pouco óbvias para conservação da biodiversidade em paisagens fragmentadas. Natureza \& Conservação, 4(2), 11-23.

Minerais do Paraná - MINEROPAR (2006). Mapa Geológico do Paraná. Recuperado em 24 de julho 2019, de http://www.mineropar.pr.gov.br/arquivos/File/2_Geral/Geologia/PDF_Mapa_Geo_650000/Mapa_Ge ologico_PR_650000_2006.pdf

Miranda, T. L. G. (2009). Plano ambiental de conservação e uso do entorno de reservatório artificial UHE Governador Bento Munhoz da Rocha Neto. LACTEC,

Morellato, L. P. C., \& Haddad, C. F. B. (2000). Introduction: The brazilian atlantic forest. Biotropica, 32(4b), 786-792. http://dx.doi.org/10.1111/j.1744-7429.2000.tb00618.x.

Murcia, C. (1995). Edge effects in fragmented forests: implications for conservation. Trends in Ecology \& Evolution, 10(2), 58-62. PMid:21236953. http://dx.doi.org/10.1016/S0169-5347(00)88977-6.

Ouyang, W., Hao, F. H., Zhao, C., \& Lin, C. (2010). Vegetation response to 30 years hydropower cascade exploitation in upper stream of Yellow River. Communications in Nonlinear Science and Numerical Simulation, 15(7), 1928-1941. http://dx.doi.org/10.1016/j.cnsns.2009.07.021.

Parrotta, J. A., Turnbull, J. W., \& Jones, N. (1997). Catalyzing native forest regeneration on degraded tropical lands. Forest Ecology and Management, 99(1-2), 1-7. http://dx.doi.org/10.1016/S03781127(97)00190-4.

Pereira, T. K., Galvão, F., Moro, R. S., Lingnau, C., \& Ferraz, S. F. B. (2017). Dinâmica da paisagem ripária do rio Pitangui no Primeiro Planalto Paranaense entre 1953 e 2012. Scientia Forestalis, 45(115), 551 562. http://dx.doi.org/10.18671/scifor.v45n115.12. 
Pirovani, D. B., Silva, A. G., Santos, A. R., Cecílio, R. A., Gleriani, J. M., \& Martins, S. V. (2014). Análise espacial de fragmentos florestais na Bacia do Rio Itapemirim, ES. Revista Árvore, 38(2), 271-281. http://dx.doi.org/10.1590/S0100-67622014000200007.

Programa das Nações Unidas para o Desenvolvimento - PNUD. (2019). Desenvolvimento Humano e IDH. Recuperado em 24 de julho 2019, de http://www.br.undp.org/content/brazil/pt/home/idh0.

R Development Core Team (2017). R: A languageand environment for statistical computing. Recuperado em 24 de julho 2019, de http://www.r-project.org

Ribeiro, M. C., Metzger, J. P., Martensen, A. C., Ponzoni, F. J., \& Hirota, M. M. (2009). The Brazilian Atlantic Forest: How much is left, and how is the remaining forest distributed? Implications for conservation. Biological Conservation, 142(6), 1141-1153. http://dx.doi.org/10.1016/j.biocon.2009.02.021.

Ricketts, T. H. (2001). The Matrix Matters: Effective isolation in fragmented landscapes. American Naturalist, 158(1), 87-99. PMid:18707317. http://dx.doi.org/10.1086/320863.

Salati, E., \& Nobre, C. A. (1992). Possible climatic impacts of tropical deforestation. In N. Myers (Ed.), Tropical Forests and Climate (pp. 177-196). Dordrecht: Springer Netherlands.

Silva, L. G., Santos, S., \& Moraes, F. (2014). Fragmentação da mata atlântica de interior: análise de paisagem do corredor verde sul-americano e florestas do alto Paraná. Boletín Geográfico (Neuquén), 32(3), 61-68. http://dx.doi.org/10.4025/bolgeogr.v32i3.21881.

Simas, M., \& Pacca, S. (2013). Energia eólica, geração de empregos e desenvolvimento sustentável. Estudos Avançados, 27(77), 97-116. http://dx.doi.org/10.1590/S0103-40142013000100008.

Sistema Nacional de Informações sobre Recursos Hídricos - SNIRH. (2017). Portal HidroWeb. Recuperado em 19 de junho 2017, de http://www.snirh.gov.br/hidroweb

Swift Junior, L. W., Swank, W. T., Mankin, J. B., Luxmoore, R. J., \& Goldstein, R. A. (1975). Simulation of evapotranspiration and drainage from mature and clear-cut deciduous forests and young pine plantation. Water Resources Research, 11(5), 667-673. http://dx.doi.org/10.1029/WR011i005p00667.

Tabarelli, M., et al (2012). A conversão da floresta atlântica em paisagens antrópicas: lições para a conservação da diversidade biológica das florestas tropicais. Interciencia, 37(2), 88-92.

Tabarelli, M., \& Gascon, C. (2005). Lições da pesquisa sobre fragmentação: aperfeiçoando políticas e diretrizes de manejo para a conservação da biodiversidade. Megadiversidade, 1(1), 181-188.

Tischendorf, L., \& Fahrig, L. (2000). On the usage and measurement of landscape connectivity. Oikos, 90(1), 7-19. http://dx.doi.org/10.1034/j.1600-0706.2000.900102.x.

Tranvik, L. J., Downing, J. A., Cotner, J. B., Loiselle, S. A., Striegl, R. G., Ballatore, T. J., Dillon, P., Finlay, K., Fortino, K., Knoll, L. B., Kortelainen, P. L., Kutser, T., Larsen, S., Laurion, I., Leech, D. M., McCallister, S. L., McKnight, D. M., Melack, J. M., Overholt, E., Porter, J. A., Prairie, Y., Renwick, W. H., Roland, F., Sherman, B. S., Schindler, D. W., Sobek, S., Tremblay, A., Vanni, M. J., Verschoor, A. M., von Wachenfeldt, E., \& Weyhenmeyer, G. A. (2009). Lakes and reservoirs as regulators of carbon cycling and climate. Limnology and Oceanography, 54(6), 2298-2314.

Trevisan, D. P., \& Moschini, L. E. (2015). Dinâmica de uso e cobertura da terra em paisagem no interior do Estado de São Paulo: Subsídios para o planejamento. Fronteiras: Journal of Social Technological and Environmental Science, 4(3), 16-30. http://dx.doi.org/10.21664/2238-8869.2015v4i3.p16-30.

United States Geological Survey - USGS (2018). Earth Explorer. Recuperado em 11 de janeiro de 2017, de https://earthexplorer.usgs.gov/

Vidolin, G. P., Biondi, D., \& Wandembruck, A. (2011). Análise da estrutura da paisagem de um remanescente de floresta com Araucária, Paraná, Brasil. Revista Árvore, 35(3), 515-525. http://dx.doi.org/10.1590/S0100-67622011000300014.

Williamson, C. E., Saros, J. E., Vincent, W. F., \& Smol, J. P. (2009). Lakes and reservoirs as sentinels, integrators, and regulators of climate change. Limnology and Oceanography, 54(6 part 2), 2273-2282.

Winjum, J. K., \& Schroeder, P. E. (1997). Forest plantations of the world: their extent, ecological attributes, and carbon storage. Agricultural and Forest Meteorology, 84(1-2), 153-167. http://dx.doi.org/10.1016/S0168-1923(96)02383-0.

Wu, J., Huang, J., Han, X., Xie, Z., \& Gao, X. (2003). Three-Gorges Dam - experiment in habitat fragmentation? Science, 300(5623), 1239-1240. PMid:12764179. http://dx.doi.org/10.1126/science.1083312.

Zaú, A. S. (1998). Fragmentação da Mata Atlântica: aspectos teóricos. Floresta e Ambiente, 5(1), 160-170. 
Zhao, Q., Liu, S., Deng, L., Dong, S., Cong, Wang, Yang, Z., \& Yang, J. (2012). Landscape change and hydrologic alteration associated with dam construction. International Journal of Applied Earth Observation and Geoinformation, 16(1), 17-26. http://dx.doi.org/10.1016/j.jag.2011.11.009.

Contribuição dos autores: AKM: conceituação, curadoria de dados, análise formal, investigação, escrita primeira redação, escrita - revisão e edição; FG: conceituação, supervisão, escrita - revisão e edição; RAMM: curadoria de dados, análise formal, escrita - primeira redação; CTB: conceituação, supervisão, escrita - revisão e edição; PCB e KGM: supervisão, escrita - revisão e edição. 